\title{
Normative and perceived unmet mental health needs, health care use and barriers to care for mental health problems in a general population sample
}

\author{
Eva Rens ( $\nabla$ eva.rens@uantwerpen.be ) \\ University of Antwerp \\ Joris Michielsen \\ Institute of Tropical Medicine (ITM) \\ Geert Dom \\ University of Antwerp \\ Roy Remmen \\ University of Antwerp \\ Kris Van den Broeck \\ University of Antwerp
}

\section{Research Article}

Keywords: Public mental health, mental health needs, unmet need, perceived need, epidemiology, treatment gap, health care use, barriers

Posted Date: February 14th, 2022

DOI: https://doi.org/10.21203/rs.3.rs-1327032/v1

License: (c) (i) This work is licensed under a Creative Commons Attribution 4.0 International License.

Read Full License 


\section{Abstract}

Background: Mental health problems often remain undetected and untreated. Prior research suggests that this is mainly due to a lack of need-perception and attitudinal barriers. The aim of this study is to examine unmet mental health needs using both a normative (assessed using screening scales) and a self-perceived approach.

Methods: A cross-sectional survey study with a weighted and representative sample of 1208 individuals aged $15-80$ years old was carried out in 2021 in the province of Antwerp (Belgium). The questionnaire consisted of screening scales for depression (PHQ-9), anxiety (GAD-7) and alcohol abuse (AUDIT-C and CAGE), and questions about dysfunction in daily life, 12-month health care use for mental health problems, self-perceived unmet mental health needs and reasons for not seeking (extra) help. Logistic regression analyses were used to explore the predictors of mental health problems, health care use, and objective and subjective unmet mental health needs.

Results: One in five respondents had a probable mental health problem according to screening scales. Among those, only one in three used health care for their mental health, resulting in a population prevalence of $14 \%$ objective unmet mental health needs. Likewise, $14 \%$ of the total sample perceived an unmet mental health need. However, more women and younger people perceived unmet needs, while normative unmet needs were higher among men and older people. One in six of the total sample used health care for their mental health, half of whom did not have a mental health problem according to the screening scales. Motivational reasons were most often endorsed for not seeking any help, while a financial barrier was the most important reason for not seeking extra help.

Conclusions: The prevalence of unmet mental health needs is high. Normative and perceived (unmet) mental health needs are both relevant and complementary, but are predicted by different factors. More research is needed on this discrepancy.

\section{Background}

Public mental health looks into mental health problems at the population level. Besides mental health promotion and prevention of mental disorders, one of the main aims of public mental health policies is to reduce mental health inequalities (1). Mental health inequalities in the population are often described in terms of unmet mental health needs. An unmet mental health need is present when someone has a mental health problem but does not seek or receive mental health care (2-5). Classically, this is operationalized as having a mental disorder, as assessed with validated instruments or a diagnostic assessment, in combination with the absence of any formal health care use for mental health related reasons (2). For example, in the European Study of the Epidemiology of Mental Disorders (ESEMeD) in the early 2000 s, it was found that about half (48\%) of those with a disabling 12 -month mental disorder reported not using any type of formal health care and only one in four $(25 \%)$ reported seeing a mental health specialist in the 12 months prior to the interview (2). At population level, $3 \%$ of adults in Europe 
have an unmet mental health need. Moreover, Demyttenaere et al. (2004) estimated that at least half of serious cases receive no treatment while the majority of people in treatment are subthreshold cases (5).

A shortcoming of 'normative' approaches (i.e., approaches using validated instruments with population norm scores to distinguish cases from non-cases) is that they do not take the subjective perception of the individual into account, while perceiving a need for mental health care is a major explanatory factor of help seeking (6-8). Olsson et al. (2020) have therefore proposed an extended definition of unmet mental health needs, in which unmet needs can occur at three stages of the pathway to adequate care: 1) not perceiving a need for care, 2) not seeking care, and 3) not receiving (sufficient or adequate) care (9). Applying this definition in a Swedish population sample, they found that more than one in three $(36 \%)$ had perceived a need for mental health care at any time in life (9). Among these 'need-perceivers', 71\% sought care, and one in four care-seekers did not experience the care as sufficient (9).

In Europe, $9 \%$ percent of the general population and 33\% of those with a mental disorder perceived some need for mental health care (10). Among those with a disabling 12-month mental disorder, $82 \%$ of those with a perceived need as compared to $11 \%$ of those without a perceived need used some kind of professional help, highlighting the relevance of need perception as a major predictor of help-seeking behavior (10). In the US, a total of $6 \%$ adults reported they felt a 'perceived need for mental health care in the past year (2018) that was not received' (11).

Some studies have used the more extensive Perceived Need for Care Questionnaire (PNCQ) which assesses whether mental health needs are fully met, partially met or unmet for specific healthcare services (12). Using the PNCQ, it was found that only a minority of all mental health needs of Dutch and Australian people with a mental disorder are fully met (13). In a Canadian general population sample, it was found that $18 \%$ reported that all their care needs were unmet, and this was especially the case for counselling needs (14).

Besides not perceiving a need for care, several other barriers to mental health care exist. Previous research has shown that attitudinal and motivational barriers, such as preferring to manage problem on one's own, are more often reported than structural barriers such as the availability of services $(4,7,15)$. The cost of services is a particularly important barrier in the US, as compared to European countries with universal health coverage (15).

In the present cross-sectional study, the prevalence and predictors of mental health problems, normative and self-perceived needs for mental health care as well as health care use for mental health problems and barriers to care are investigated in the province of Antwerp (Belgium). Prior explorative qualitative research in the region suggested that unmet mental health needs are high, especially among people living in poverty, ethnic minorities, and in the young and oldest age groups (16). We use the term normative needs when (unmet) mental health needs are assessed using normative criteria and are thus measured using 'objective' validated instruments. The term subjective or perceived (unmet) needs is used for selfperceived mental health needs as reported by the individual. 


\section{Methods}

\section{Design and survey sample}

The study is part of a research project which aims to assess the (unmet) mental health needs of the Antwerp Province in Flanders, Belgium. The study was carried out in one rural and one urban primary care zone (PCZ). PCZs are regional structures consisting of approximately 100000 inhabitants and designed to improve the collaboration between local authorities and care providers. The rural PCZ (the region of $\mathrm{Mol}$ ) has a less extensive range of mental health care in the area, while the urban PCZ (the east region of Antwerp city) is highly multicultural and has a more extensive range of mental healthcare.

A sample of 5000 inhabitants aged 15 to 80 years was invited to participate in a mental health survey. The sample was randomly drawn from the national register and was stratified by gender, municipality, age and nationality (Belgian versus non-Belgian). Non-Belgians were oversampled as a lower response rate was expected in this group, based on previous research. Invited individuals received two postal invitations between May and July 2021. The first letter included a link and QR-code to the online questionnaire, and the second invitation also included a paper questionnaire that could be returned free of charge. The online questionnaire was available in six languages: Dutch, French, English, German, Polish and Arabic. The questionnaire consisted of 94 questions in total, but the vast majority of participants did not have to answer all questions due to skip logic. Forced response was implemented in the online questionnaire to avoid missing data, but participants could still indicate 'I don't know' or 'not applicable' on most questions. The completion time was estimated at ten minutes. Informed consent was obtained from all subjects and/or their legal guardian.All methods were carried out in accordance with relevant guidelines and regulations.

\section{Variables and instruments}

The questionnaire was fully self-report and consisted of socio-demographic questions, screening scales for common mental health problems, and questions about perceived unmet mental health needs.

Depending on the given answers, additional questions about the number and type of professional health care providers which were consulted for mental health problems or the barriers to care were presented.

The following socio-demographic information was included in the current study: age category $(15-25 y$ old, 26 - 39y old, $40-64 y$ old, $65-80 y$ old), gender (M, F), origin (geographic region of Europe, nonEurope), educational attainment (primary education, secondary education, higher education i.e. college or university), financial distress (self-reported financial difficulties or not) and urbanicity of the residence (urban, rural).

\section{Mental health problems}


Short screening questionnaires were used for depression, anxiety disorder and alcohol disorder. The presence of depression was assessed using the Patient Health Questionnaire-9 (PHQ-9 (17)) and the diagnostic DSM-IV algorithm was used to distinguish people with and without any type of probable depressive disorder (both Major Depressive and Other Depressive Syndrome). This scoring method was chosen as it yields a higher specificity, but the 'other depressive syndrome' scoring (positive screening when at least two symptoms are indicated at least at more than half the days and one of the symptoms is depressed mood or anhedonia) was included as well to compensate for lower sensitivity (18).

The presence of clinical anxiety was assessed using the Generalized Anxiety Disorder-7 (GAD-7 (19)), which has good validity and reliability for screening in the general population (20). A score of 10 or higher indicates the presence of a probable generalized anxiety disorder.

Two short questionnaires were used to assess alcohol abuse. The AUDIT-C assesses frequencies and quantities of alcohol consumption, and a cut-off point of $\geq 5$ for men and $\geq 4$ for women was used (21). The four CAGE questions examined alcoholism and a score of $\geq 2$ was used as a cut-off point (22). The presence of an alcohol disorder was defined as a positive screening on both the AUDIT-C and the CAGE.

Finally, a variable was constructed indicating the presence of any mental health problem, which means someone has at least one probable disorder of the ones assessed (= positive screening for depressive disorder and/or anxiety disorder and/or alcohol disorder). Comorbidities were not assessed.

Dysfunction in daily life due to psychological problems was examined as well. All respondents indicated the extent to which their social and leisure life, work or study, and family life were affected by psychological problems on a scale from zero to ten. A score of at least six on one of the scales was considered a significant level of dysfunction.

\section{Health care use for mental health problems}

All participants answering 'yes' on the question "In the past 12 months, have you been in contact with a professional care provider (e.g., general practitioner, psychologist,...) because of psychological problems, your emotions, or alcohol or drug use?" were asked to specify whether they had contact with a general practitioner, a psychologist or psychotherapist, a psychiatrist, and whether they were prescribed medication for mental health problems.

\section{Normative unmet mental health needs}

A normative unmet mental health need is defined as the presence of any mental health problem as assessed by screening scales together with the absence of health care use for mental health problems in the past 12 months. No distinction was made between the type of care provider or the number of contacts. 


\section{Perceived unmet mental health needs and barriers}

Participants without any twelve-month professional contact for mental health problems were asked "During the past 12 months, have you thought you might need help for psychological problems, your emotional problems, or alcohol or drug use?". Those replying 'yes', are considered those with 'perceived fully unmet needs', and those replying 'no' as those with 'no perceived need'. On the other hand, participants reporting health care use for mental health problems in the past twelve months were asked whether they thought the received care was sufficient. Respondents replying with 'yes', are considered as those with 'met needs', whereas those replying with 'no' are considered as those with 'perceived partially unmet needs'.

All subjects who responded that they thought they might have needed help but did not seek it, or that they did not receive sufficient help, were asked to endorse all the reasons that were applicable from a list of nine reasons, or could specify a reason themselves.

\section{Statistical analyses}

The individual observations were weighed using inverse probability weighting to correct for differences in response rate between age categories, gender and nationality. Missing data were very low $(<1 \%$ for every variable) because of the forced response implementation in the online questionnaire, and therefore it was decided to use available case analysis. Participants who returned the paper questionnaire and had a high level of missing data or missing demographic information were excluded from the analysis $(n=14)$. All analyses were conducted in IBM SPSS Statistics version 28.

Descriptive characteristics of the sample and the prevalence of mental health needs, health care use for mental health problems, perceived and normative unmet mental health needs, and barriers to care are reported using weighted percentages.

Logistic regression analyses were carried out to assess the likelihood of having a mental health problem, using health services for mental health problems, having a normative unmet mental health need, and having a perceived unmet mental health need. The logistic regression models of the presence of a mental health problem, health care use and perceived unmet needs consider the full sample, while the logistic regression modelling normative unmet needs considers only those with any mental health problem. All multivariable models include all sociodemographic factors. The multivariable models of health care use and perceived unmet needs also include the presence of any mental health need and dysfunction, and the multivariable model of normative unmet needs includes dysfunction on top of the sociodemographic factors.

Unadjusted odds ratios (UOR) and adjusted odds ratios (AOR) are reported with their $95 \%$ confidence intervals $(\mathrm{Cl})$. P-values of the comparisons to the reference and the overall significance of the factor in the 
multivariable model are indicated by asterisks. Interactions were not included. A significance level of $p<$ .05 was established for all analyses.

\section{Results}

\section{Participants}

A total of 1208 people (24.2\% response rate) fully participated, most of them $(79.2 \%)$ online. The vast majority (93.5\%) completed the questionnaire in Dutch, 3.2\% in English, 1.4\% in French, 1.3\% in Arabic and $0.5 \%$ in Polish. Non-response was higher among men, younger people and people with a birthplace outside Europe.

In the weighted sample, $49.8 \%$ were women. The mean age was 45.5 years old (SD $=17.8$ ), with $16.5 \%$ people aged 25 or younger, $32.4 \%$ aged between 26 and 44 years old, $33.7 \%$ aged between 45 and 64 years old and $17.3 \%$ aged 65 or older. One in nine (11.1\%) participants was born outside of Europe, and $55.6 \%$ lived in an urban residence. As regards education, $13.9 \%$ did not have a secondary education degree and $41.1 \%$ has a higher education degree. More than one in six $(17.5 \%)$ reported financial distress in the past twelve months.

\section{Prevalence and predictors of mental health problems}

Approximately one in five (21.5\%) has a mental health problem as assessed by screening questionnaires: $10.2 \%$ had a probable depressive disorder, $10.0 \%$ a probable anxiety disorder, and $8.8 \%$ a probable alcohol disorder. A total of $20.3 \%$ of the sample experiences significant dysfunction in daily life due to psychological problems, but this does not completely overlap with the group of people with a mental health need as assessed by the screening questionnaires. Approximately half $(49.6 \%)$ of those with a mental health problem experienced dysfunction in daily life, as opposed to $12.4 \%$ of those who did not have a positive screening on one of the mental health scales.

Logistic regression analysis was used to assess the likelihood of having a mental health problem (Table 1). There was no significant gender effect. Age is a significant predictor of having any mental health need, such that younger age groups have a higher prevalence of mental health problems as compared to older people. Specifically, people aged 65 or older had a lower likelihood of having a mental health problem as compared to people aged 25 or less $(\mathrm{OR}=.39,95 \% \mathrm{Cl}=.22-.70)$. Compared to people with a

primary education degree, the likelihood of having a mental health problem was lower among people with a secondary education degree $(\mathrm{OR}=.61,95 \% \mathrm{Cl}=.40-.95)$ or a higher education degree $(\mathrm{OR}=.56,95 \% \mathrm{Cl}$ $=.35-.90)$. Financial distress was strongly predictive of mental health problems $(\mathrm{OR}=3.44,95 \% \mathrm{Cl}=$ 2.42 - 4.89). Finally, mental health problems were more common among people with a non-European birthplace and people living in an urban residence, but these effects are not significant when other factors are taken into account. 


\section{Prevalence and predictors of health care use for mental health problems}

Considering the total sample, one in six (17.6\%) reported health care use for mental health problems in the past twelve months. Psychologists (11.4\%) and GPs (11.3\%) were more often consulted compared to psychiatrists (3.6\%), and $6.4 \%$ was prescribed medication for mental health problems. Results of the logistic regression analysis examining the predictors of health care use for mental health problems in the general population are shown in Table 2. A significant difference in the likelihood of using health services for mental health problems was found, with $21.4 \%$ women as compared to $12.9 \%$ men consulting a health professional for their mental health $(\mathrm{OR}=2.08,95 \% \mathrm{Cl}=1.47-2.94)$. Age is a significant predictor of health care use, with people aged 65 and older being less likely to consult a professional for mental health problems compared to the reference of $15-25$-year olds $(\mathrm{OR}=.43,95 \% \mathrm{Cl}=.19-.96)$. Individuals with a non-European origin were less likely to use health services for their mental health in the multivariable model only $(\mathrm{OR}=.39,95 \% \mathrm{Cl}=.21-.73)$. In contrast, individuals with financial distress were significantly more likely to consult a health care professional for mental health problems in crude analysis, but this predictor was not significant in the multivariable model.

Besides the sociodemographic predictors, also the presence of a mental health problem and dysfunction were included to adjust for differences in mental health needs. As expected, the presence of a positive screening for a mental health problem $(\mathrm{OR}=2.62,95 \% \mathrm{Cl}=1.79-3.84)$ and dysfunction $(\mathrm{OR}=4.70,95 \%$ $\mathrm{Cl}=3.21-6.88$ ) are highly predictive of using health care for mental health problems. The effect is largest for the presence of dysfunction, with $10.5 \%$ of those without as compared with $43.8 \%$ of those with functional problems using health services for mental health problems.

\section{Prevalence and predictors of normative unmet mental health needs}

The classification and population distribution of normative unmet mental health needs is shown in Figure 1. Among participants with any mental health problem, only $36.7 \%$ used health care for their mental health. Although this is significantly more than the $11.8 \%$ of those without a mental health need $\left(X^{2}=\right.$ $88.68, \mathrm{df}=1, \mathrm{p}<.01$ ), only $45.9 \%$ of all people who used health services for mental health reasons has a mental health problem according to the screening questionnaires. In the total sample, $13.6 \%$ presents a normative unmet need for mental health care.

Table 3 shows the distribution and logistic regression analysis of having a normative unmet mental health need. Note that both the percentage within the category as a whole is shown, as well as the percentage within the category with a mental health need, as the former is highly dependent on the prevalence of mental health problems in that group. The logistic regression results therefore apply to the subsample of people with any mental health need $(\mathrm{N}=259)$. An estimated $15.7 \%$ of all men have a normative unmet need as compared to $11.5 \%$ of all women. Among women with a mental health need, 
$55.6 \%$ did not use health services, which is significantly less than the $70.3 \%$ men with a mental health need who did not use health services $(\mathrm{OR}=.56,95 \% \mathrm{Cl}=.32-.98)$. Age is not a significant predictor in the multivariable model, but the 65+ age group differs significantly from the youngest age group $(\mathrm{OR}=7.21$, $95 \% \mathrm{Cl}=1.11-46.64)$. This finding should be interpreted with caution because of the low prevalence of mental health needs in the oldest age group, leading to very wide confidence intervals in this group. The level of unmet mental health needs is rather low among the total group of people aged 65 and older $(9.9 \%)$ due to the low prevalence of mental health needs, but remarkably high in the subgroup of older adults with a mental health need $(90.7 \%)$. Individuals with a mental health need experiencing financial distress are significantly less likely to have a normative unmet mental health need as compared to those without financial distress $(\mathrm{OR}=.51,95 \% \mathrm{Cl}=.27-.95)$. Finally, the presence of dysfunction due to mental health problems is associated with a lower likelihood of having a normative unmet mental health need $(\mathrm{OR}=.42,95 \% \mathrm{Cl}=.23-.75)$.

\section{Table 3}

Logistic regression analysis modelling the likelihood of having a normative unmet need, i.e., not using health care for mental health among participants with a mental health problem $(N=259)$. 
Any normative unmet need

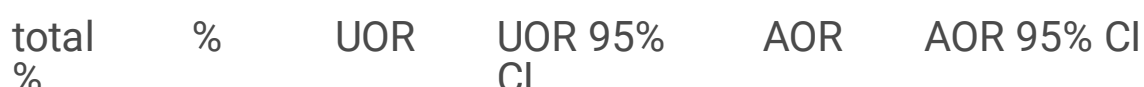

\begin{tabular}{|c|c|c|c|c|c|c|c|}
\hline Gender* & Male (ref.) & 15.7 & 70.3 & & & & \\
\hline & Female & 11.5 & 55.6 & $.53^{*}$ & $.32-.88$ & $.56^{\star}$ & $.32-.98$ \\
\hline \multirow[t]{4}{*}{ Age } & $15-25$ (ref.) & 16.8 & 66.0 & & & & \\
\hline & $26-44$ & 16.3 & 57.9 & .71 & $.36-1.42$ & .69 & $.30-1.62$ \\
\hline & $45-64$ & 11.2 & 60.7 & .80 & $.38-1.67$ & .76 & $.31-1.82$ \\
\hline & $65-80$ & 9.9 & 90.7 & $5.02^{\star}$ & $1.10-23.0$ & $7.21^{\star}$ & $\begin{array}{l}1.11- \\
46.64\end{array}$ \\
\hline \multirow[t]{3}{*}{ Education } & $\begin{array}{l}\text { Primary } \\
\text { (ref.) }\end{array}$ & 21.1 & 65.8 & & & & \\
\hline & Secondary & 14.1 & 67.9 & 1.10 & $.55-2.19$ & 1.02 & $.45-2.30$ \\
\hline & Higher & 10.8 & 56.8 & .68 & $.34-1.37$ & .57 & $.23-1.39$ \\
\hline \multirow{2}{*}{$\begin{array}{l}\text { Financial } \\
\text { distress* }\end{array}$} & No (ref) & 11.6 & 68.2 & & & & \\
\hline & Yes & 22.8 & 53.9 & $0.55^{\star}$ & $.33-.93$ & $.51 *$ & $.27-.95$ \\
\hline \multirow[t]{2}{*}{ Birthplace } & Europe (ref.) & 13.0 & 63.4 & & & & \\
\hline & Non-Europe & 17.9 & 62.0 & .94 & $.47-1.91$ & 1.28 & $.55-2.98$ \\
\hline \multirow[t]{2}{*}{ Urbanicity } & Urban (ref.) & 14.7 & 61.2 & & & & \\
\hline & Rural & 12.1 & 66.5 & 1.26 & $.75-2.14$ & .99 & $.55-1.80$ \\
\hline \multirow[t]{2}{*}{ Dysfunction** } & No & 9.8 & 72.7 & & & & \\
\hline & Yes & 27.3 & 52.4 & $.42^{\star \star}$ & $.25-.70$ & $.42^{\star *}$ & $.23-.75$ \\
\hline
\end{tabular}

UOR $=$ Unadjusted Odds Ratio

AOR $=$ Adjusted Odds Ratio (adjusted for all variables)

$* \mathrm{p}<.05 \quad * * \mathrm{p}<.01 \quad * * * \mathrm{p}<.001$

Asterisks after the variable name represent significance of the factor in the multivariable model. 


\section{Prevalence and predictors of perceived unmet mental health needs}

Figure 2 shows the classification table and population distribution of perceived unmet needs. One in ten $(10.0 \%)$ indicated they felt a need for mental health care but did not seek or receive help ('perceived fully unmet needs'). Among the care-seekers, the majority (76.6\%) thought the help was sufficient, resulting in $4.0 \%$ 'perceived partially unmet needs' in the population. Altogether, $14.0 \%$ of the population perceives an unmet mental health need.

Predictors of perceiving an unmet need for mental health care (both partially and fully) were assessed using logistic regression analysis, shown in Table 4. Women were more likely to perceive an unmet need for mental health care $(\mathrm{OR}=1.80,85 \% \mathrm{Cl}=1.24-2.61)$. Age was a significant predictor of perceived unmet needs, as there is a trend towards more perceived unmet needs in the youngest age groups as compared to the oldest age groups. For example, $20.7 \%$ of the $26-44$-year-olds perceived an unmet mental health need, in contrast to $11.0 \%$ of $45-64$-year-olds and $2.8 \%$ of participants aged 65 and older. In the multivariable model, individuals aged 65 and older are significantly less likely to perceive an unmet mental health need as compared to the youngest age group $(\mathrm{OR}=.19,95 \% \mathrm{Cl}=.07-.53)$. Individuals experiencing financial distress have a higher likelihood to perceive an unmet mental health need (OR = $1.79,95 \% \mathrm{Cl}=1.13-2.84)$. Individuals born outside Europe are significantly less likely to perceive an unmet mental health need $(\mathrm{OR}=.51,95 \% \mathrm{Cl}=.27-.96)$.

The presence of a mental health need and dysfunction were taken into account as well. Approximately one in ten individuals without a mental health problem perceived an unmet mental health need, compared to one in three individuals with a mental health problem, making the presence of a mental health need highly predictive of perceived unmet needs $(\mathrm{OR}=2.30,95 \% \mathrm{Cl}=1.54-3.44)$. This effect is even stronger for the presence of dysfunction: $38.0 \%$ of individuals with and $8.1 \%$ individuals without dysfunction in daily life perceived an unmet mental health need $(\mathrm{OR}=4.21,95 \% \mathrm{Cl}=2.82-6.28)$.

Table 4.

Logistic regression analysis modelling the likelihood of perceiving a fully or partially unmet need for mental health care in the general population $(N=1208)$. 
Any perceived unmet need

\begin{tabular}{|c|c|c|c|c|c|c|}
\hline & & $\%$ & UOR & UOR $95 \% \mathrm{Cl}$ & AOR & AOR $95 \% \mathrm{Cl}$ \\
\hline \multirow[t]{2}{*}{ Gender** } & Male (ref.) & 11.2 & & & & \\
\hline & Female & 16.8 & $1.61^{\star \star}$ & $1.15-2.24$ & $1.80^{\star *}$ & $1.24-2.61$ \\
\hline \multirow[t]{4}{*}{ Age $^{\star \star \star}$} & $15-25$ (ref.) & 18.8 & & & & \\
\hline & $26-44$ & 20.7 & 1.13 & $.73-1.74$ & 1.27 & $.73-2.19$ \\
\hline & $45-64$ & 11.0 & $.54^{\star \star}$ & $.33-.86$ & .72 & $.41-1.28$ \\
\hline & $65-80$ & 2.8 & $.13^{\star \star \star}$ & $.05-.31$ & $.19 \star \star$ & $.07-.53$ \\
\hline \multirow[t]{3}{*}{ Education } & Primary (ref.) & 16.5 & & & & \\
\hline & Secondary & 11.6 & .66 & $.41-1.08$ & .85 & $.47-1.55$ \\
\hline & Higher & 15.8 & .95 & $.59-1.53$ & 1.20 & $.64-2.26$ \\
\hline \multirow[t]{2}{*}{ Financial distress* } & No (ref) & 12.0 & & & & \\
\hline & Yes & 22.9 & 2.17 & $1.50-3.16$ & $1.79 *$ & $1.13-2.84$ \\
\hline \multirow[t]{2}{*}{ Birthplace* } & Europe (ref.) & 14.0 & & & & \\
\hline & Non-Europe & 13.7 & .98 & $.58-1.64$ & $.51 *$ & $.27-.96$ \\
\hline \multirow[t]{2}{*}{ Urbanicity } & Urban (ref.) & 15.4 & & & & \\
\hline & Rural & 12.2 & .76 & $.55-1.06$ & .91 & $.62-1.34$ \\
\hline \multirow[t]{2}{*}{ 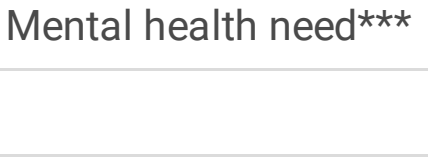 } & No & 9.5 & & & & \\
\hline & Yes & 30.8 & $4.25^{\star \star \star}$ & $3.02-5.98$ & 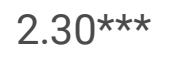 & $1.54-3.44$ \\
\hline \multirow[t]{2}{*}{ 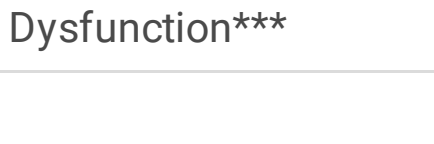 } & No & 8.1 & & & & \\
\hline & Yes & 38.0 & 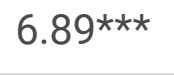 & $4.87-9.77$ & $4.21^{\star \star}$ & $2.82-6.28$ \\
\hline
\end{tabular}

UOR $=$ Unadjusted Odds Ratio

AOR $=$ Adjusted Odds Ratio (adjusted for all variables)

$* \mathrm{p}<.05 * * \mathrm{p}<.01 \quad * * * \mathrm{p}<.001$

Asterisks after the variable name represent significance of the factor in the multivariable model

\section{Barriers to mental health care}


Participants who perceived an unmet mental health need were asked to indicate which reasons explain why they did not seek or receive (sufficient) help. The prevalence of the reported barriers to mental health care is shown in Table 5. For those who did not seek care, the most often cited reason is that they prefer to handle problems on their own (65.6\%), followed by thinking it wouldn't help (30.1\%) and time constraints $(27.5 \%)$. One in four $(24.5 \%)$ reported the cost as a barrier. For those with partially unmet needs, cost was the most often cited barrier (43.0\%), followed by a preference to handle problems on their own (38.6\%). Both among those with fully unmet needs and those with partially unmet needs, about one in five people reported other barriers, for example, an expected waiting time and bad experiences in the past.

\section{Table 5}

Prevalence of endorsed reasons for not seeking or receiving (extra) help among participants who perceived an unmet need for mental health care.

\begin{tabular}{|lll|}
\hline Reason & $\begin{array}{l}\text { Fully unmet need } \\
(\mathrm{N}=121)\end{array}$ & Partially unmet need (N=48) \\
\hline I prefer to handle problems on my own & $65.6 \%$ & $38.6 \%$ \\
\hline I don't think it would help & $30.1 \%$ & $24.1 \%$ \\
\hline I don't have time for it & $27.5 \%$ & $9.0 \%$ \\
\hline I worry about the costs & $24.5 \%$ & $43.0 \%$ \\
\hline I don't know where to go for (extra) help & $21.8 \%$ & $26.3 \%$ \\
\hline I'm afraid others would think bad of me & $10.7 \%$ & $22.5 \%$ \\
\hline I don't speak the language well & $6.7 \%$ & $7.1 \%$ \\
\hline I asked for it, but didn't get (extra) help & $2.3 \%$ & $16.0 \%$ \\
\hline I cannot get there (e.g. no transport) & $1.9 \%$ & $4.8 \%$ \\
\hline Another reason & $20.4 \%$ & $20.1 \%$ \\
\hline
\end{tabular}

\section{Discussion}

This cross-sectional survey study evaluated common mental health needs (depression, anxiety and alcohol problems) in a representative general population sample in Antwerp, Belgium. A total of 1208 people aged 15 to 80 years old participated in the study. It was found that about one in five $(22 \%)$ has a probable depressive disorder, anxiety disorder or alcohol disorder. Mental disorders were found to be more common among younger age groups, people with a lower education level and people with financial problems. As expected, the presence of mental problems is linked to dysfunction in daily life. In total, our data suggest that a fifth of all individuals suffers from functional problems. This concerns half of the 
people with a mental health need, but surprisingly, also $12 \%$ of those without a positive screening on one of the screening scales indicate that their daily life is at least moderately impacted by psychological problems.

Furthermore, only about one third of people with a probable mental disorder consulted a health care professional for their mental health, resulting in a population prevalence of $14 \%$ normative unmet mental health needs. In addition, $14 \%$ of the population perceived an unmet mental need themselves, although the predictors of normative and perceived unmet mental health needs differ considerably.

Overall, one in six people discussed mental health related issues with a health care professional within the past year, especially with a psychologist and/or GP. Men, people aged 65 and older, and people born outside of Europe were less likely to use health care for their mental health. Health care use for mental health problems in the general population is higher than generally reported in other studies, where approximately one in ten people use formal health services for their mental health (23-25). This may be due to the broader definition of health care use, namely any contact with a health professional for mental health reasons (incl. emotional problems or substance abuse). It was found that the ones consulting a health professional for their mental health are not necessarily the same as those with a probable mental disorder. Almost two thirds (63.3\%) of participants with a probable mental disorder did not consult a professional for their mental health. Because the quantity and quality of care were not taken into account, this approach may even lead to an underestimation of the actual unmet mental health needs. In prior research it was suggested that approximately half of treatments in high-income countries do not meet minimally adequate treatment (MAT) criteria (i.e., eight or more psychotherapy visits or four or more visits to a doctor with pharmacotherapy) (26-28).

Normative unmet mental health needs are on a population level more common among young people, but this can be explained by their higher level of mental health problems. In contrast, only $11 \%$ of people aged 65 and older have a mental health problem according to screening scales, but $90 \%$ of these cases did not receive any form of care for mental health problems. Consistent with previous research, older people with a mental disorder are less likely to seek help when needed, especially because they tend to underestimate their own needs (29-32). Also a gender difference is present, with $70 \%$ of men versus $56 \%$ of women with a probable mental disorder who did not seek help for mental health problems, but this result was insignificant in the multivariable logistic regression model.

The population proportion of people with financial problems with unmet mental health needs was higher than the population proportion of people without financial problems with unmet mental health needs because of their higher prevalence of mental health problems. Individuals with financial distress with a mental health problem more often sought help compared to people without financial distress. In line with this finding, a longitudinal study in the UK reported higher levels of treatment with medication and psychological therapy among people from disadvantaged backgrounds (33). Other studies reported an increased risk of unmet needs among people with lower income $(4,34)$, or reported no clear association $(35,36)$. Firstly, it must be noted that financial distress was self-reported in this study, and people might 
differ in the way they define financial difficulties. Secondly, people with more financial resources might have more possibilities for self-care or other alternatives such that professional help is less needed. Finally, the design doesn't allow to draw causal conclusions, and the interpretation is especially difficult because of the reciprocal relationship between mental illness and poverty (37).

Another remarkable finding is that more than half $(54 \%)$ of those who discussed mental health related problems with a professional had no current mental health need as assessed by screening questionnaires. Several reasons can account for this finding. First, these individuals may be subthreshold cases or may have a mental health problem that was not assessed in the study. Second, mental health needs were assessed at point-prevalence, while health care contacts for mental health reasons were estimated at 12-month prevalence. It may therefore be possible that some people have had a mental health problem that is already solved. Finally, this may also be an expression of 'overmet need'. Research has shown that people without a mental disorder account for a significant proportion of healthcare users, but that these individuals often have other need indicators, and generally have fewer visits and use less specialist services $(5,23,38)$. People with mental distress receiving some professional help should therefore not be regarded as having 'overmet need', as this can alleviate mild mental health problems and prevent problems from worsening.

Unmet mental health needs were also assessed from a subjective perspective. A perceived unmet mental health need is present when someone did not seek care but perceived a need for mental health care (= fully perceived unmet need), or when someone did seek care but felt that this was not sufficient (= partially perceived unmet need). In total, $14.0 \%$ perceived an unmet mental health need, of which the majority are fully unmet. When help was received, $23 \%$ felt that they were insufficiently helped. In line with previous research, men and older people were less likely to perceive an unmet need for mental health care $(9,14,39)$. Contrary to normative unmet needs, individuals experiencing financial distress more often perceived an unmet mental health need, but this can be attributed to the different sample studied (subsample with mental health problem vs. total sample).

When an unmet mental health need was perceived, participants were asked to endorse all reasons for not seeking (extra) help. As expected from the literature, the most frequently reported barriers for not getting help are motivational or attitudinal barriers $(4,15)$. Two-thirds cited self-reliance as the reason for not seeking help, and nearly a third thought it wouldn't help. A quarter of the people who did not seek help mentioned cost as a barrier. However, among individuals who received help but felt this was insufficient, financial reasons were most often endorsed. This suggests that the cost of mental health care is in Belgium primarily an obstacle to obtaining adequate care as long as needed (e.g., the majority of psychotherapy was not reimbursed at the time of data-collection).

A major advantage of the study is the public mental health perspective. Other strengths of the study are the use of a representative probability sample and the inclusive nature of the study. For example, online participation was possible in six languages including Arabic, and the wider age range allowed 15 to 80 - 
years old to participate. Finally, the study covered a rather small region, allowing local healthcare providers to match their care offer to the mental health needs in the region.

It must be noted that the data collection took place between May and August 2021, which means some covid-19 related freedom-restrictions were still implemented and may have influenced the findings. Prior research showed no statistical difference between met and unmet need for mental health care, but point estimates were suggestive of higher unmet needs among those with a current mental disorder after the lock-down period (40). Comparison with the province of Antwerp in the Belgian Health Interview survey suggests that the prevalence of mental health problems has risen substantially since 2018: probable depression rose from $6 \%$ to $10 \%$, anxiety disorder remained the same $(11 \%)$, and alcohol abuse (based on the CAGE questionnaire only) doubled from $6 \%$ to $13 \%(41,42)$. However, no comparable Antwerp data is available on perceived or unmet needs.

Additionally, though validated instruments were used, the exclusive use of symptom screening questionnaires may be considered a limitation. These measures are indicative of mental disorders but tend to overestimate the true prevalence in the population $(43,44)$. Moreover, only common mental disorders were included. Also, the disorder type and severity were not considered when studying unmet needs. However, this may be relevant, for example as previous research suggests that men may be more likely than women to delay using health care for minor mental health concerns, but that gender effects diminish when problems are more serious (45). Also, people with a substance use disorder tend to be less likely to perceive a need for care and seek treatment $(46,47)$.

As a final remark, only one quarter of the invited sample participated, despite two postal invitations and the possibility to participate online and offline. However, this response-rate was anticipated and the data were weighted to match the population distribution, also correcting for minor inequalities in non-response across strata.

An important finding is that unmet mental health needs are high, with a population prevalence of $14 \%$ for both perceived and normative unmet needs. This is remarkably higher than estimates reported elsewhere, but different definitions and operationalizations complicate comparisons $(2,4)$. However, the overlap between perceived and normative unmet needs is small. Additional analysis revealed that 116 participants have only a normative unmet need, 121 people have only a perceived unmet need, and merely 48 people ( $16.8 \%$ of those with any normative and/or perceived unmet need) have both a normative and perceived unmet need for mental health care. Moreover, unmet normative and perceived needs are explained by different factors. Especially, more women perceived an unmet need for mental health care, but more men with a probable mental disorder did not seek care. Little differences were found in unmet needs as regards to education and urbanicity. Future research should further explore the link between normative and perceived (unmet) needs and its associated factors. A combination of subjective and objective approaches allows researchers and policymakers to assess the (unmet) need for mental health care on a population level with special attention to the individual perspective. 
Further efforts should be made to make mental health care more accessible for everyone. The reported reasons for not seeking or receiving adequate care may provide guidance to policy makers. To ensure that every individual with a mental health need receives adequate care, stepped care principles should be respected such that people with mild needs are helped in generalist or primary care services, and people with more severe needs in specialist services. Information and awareness campaigns remain important to help people overcome motivational barriers to mental health care. Also financial barriers to obtaining extra help need to be addressed. Finally, the totality of health and social care needs of people with mental health problems should be addressed as well, so that not only the 'treatment gap' but the whole mental health 'care gap' can be reduced (3).

\section{Conclusion}

This cross-sectional survey study evaluated common mental health needs (depression, anxiety and alcohol problems) in a representative general population sample in Antwerp, Belgium. Both self-reported perceived unmet needs and normative mental health needs measured by validated scales were examined. One in five had a probable mental disorder and one in six participants discussed their mental health with a professional in the past year. Among those with a positive mental disorder screening, only a third had contact with a health professional for their mental health. In the general population, $14 \%$ had normative mental health need. More men, older people, and people without financial distress or functional problems had a normative unmet need.

With regard to perceived unmet needs, one in ten people thought they needed some help for their mental health but did not seek any, and $4 \%$ received some care but thought this was insufficient, resulting in a total population prevalence of $14 \%$ perceived unmet needs. As opposed to normative unmet needs, perceived unmet needs were more common among women, younger people, people with financial distress or a non-European background, and those with functional problems or a normative mental health need. Motivational and attitudinal barriers, especially the preference to handle problems on their own, are generally most often endorsed. However, the cost is mainly a barrier to obtaining extra help.

\section{Abbreviations}

ESEMeD = European Study of the Epidemiology of Mental Disorders

PNCQ $=$ Perceived Need for Care Questionnaire

$\mathrm{PCZ}=$ primary care zone

UOR = unadjusted odds ratio

$\mathrm{AOR}=$ adjusted odds ratio

$\mathrm{Cl}=$ confidence interval

Page $17 / 23$ 


\section{Declarations}

\section{Ethics approval and consent to participate}

Ethical approval was obtained on January $12^{\text {th }} 2021$ by the Ethics Committee for the Social Sciences and Humanities (EA SHW) of the University of Antwerp (file SHW_20_130). Approval for sampling from the national register was obtained on the 15th of February 2021 (Decision 15/2021). Informed consent was obtained from all subjects and/or their legal guardian. All methods were carried out in accordance with relevant guidelines and regulations.

\section{Consent for publication}

Not applicable.

\section{Availability of data and materials}

The datasets generated and/or analysed during the current study are not publicly available due to privacy regulations but are available from the corresponding author on reasonable request.

\section{Competing interests}

The authors declare that they have no competing interests.

\section{Funding}

The research is funded by the Flemish government and by the Academic Chair Public Mental Health, which is financially supported by the participating hospitals (https://www.uantwerpen.be/en/chairs/public-mental-health/partners/).

\section{Authors' contributions}

All authors are member of the Chair Public Mental Health. KVdB is holder of the Chair, GD and RR are promoters of the Chair, JM is voluntary academic staff member and ER is PhD student. ER performed the analyzes and wrote the main body of the manuscript, which was subsequently improved and supplemented by JM, GD, RR and KVdB. All authors contributed to the final manuscript. All authors read and approved the final manuscript.

\section{Acknowledgements}


The study is part of the Chair Public Mental Health at the University of Antwerp. We wish to thank the funding hospitals and Flemish government. We also wish to thank prof. dr. Hans De Loof and dr. Kirsten Catthoor for their help in the interpretation of the findings.

\section{References}

1. Wahlbeck K. Public mental health: the time is ripe for translation of evidence into practice. World Psychiatry 2015;14:36-42.

2. Alonso J, Codony $M$, Kovess $\mathrm{V}$, et al. Population level of unmet need for mental healthcare in Europe. Br J Psychiatry 2007;190:299-306.

3. Pathare S, Brazinova A, Levav I. Care gap: a comprehensive measure to quantify unmet needs in mental health. Epidemiol Psychiatr Sci 2018;27:463-7.

4. Corscadden L, Callander EJ, Topp SM. Who experiences unmet need for mental health services and what other barriers to accessing health care do they face? Findings from Australia and Canada. Int $J$ Health Plann Manage 2019;34:761-72

5. Demyttenaere K, Bruffaerts R, Posada-Villa J, et al. Prevalence, severity, and unmet need for treatment of mental disorders in the World Health Organization World Mental Health Surveys. JAMA 2004;291:2581-90.

6. Mojtabai R, Olfson M, Sampson NA, et al. Barriers to mental health treatment: results from the National Comorbidity Survey Replication. Psychol Med 2011;41:1751-61.

7. Andrade LH, Alonso J, Mneimneh Z, et al. Barriers to mental health treatment: results from the WHO World Mental Health surveys. Psychol Med 2014;44:1303-17.

8. Verhaak PF, Prins MA, Spreeuwenberg P, et al. Receiving treatment for common mental disorders. Gen Hosp Psychiatry 2009;31:46-55.

9. Olsson S, Hensing G, Burström B, Löve J. Unmet Need for Mental Healthcare in a Population Sample in Sweden: A Cross-Sectional Study of Inequalities Based on Gender, Education, and Country of Birth. Community Ment Health J 2021;57:470-81.

10. Codony $\mathrm{M}$, Alonso J, Almansa J, et al. Perceived need for mental health care and service use among adults in Western Europe: results of the ESEMeD project. Psychiatr Serv 2009;60:1051-8.

11. KFF. Adults Reporting Unmet Need for Mental Health Treatment in the Past Year, Timeframe 2018 2019. Retrieved from https://www.kff.org/other/state-indicator/adults-reporting-unmet-need-formental-health-treatment-in-the-past-year/

12. Meadows G, Harvey C, Fossey E, Burgess P. Assessing perceived need for mental health care in a community survey: development of the Perceived Need for Care Questionnaire (PNCQ). Soc Psychiatry Psychiatr Epidemiol 2000;35:427-35.

13. Prins $M$, Meadows $G$, Bobevski I, et al. Perceived need for mental health care and barriers to care in the Netherlands and Australia. Soc Psychiatry Psychiatr Epidemiol 2011;46:1033-44. 
14. Fleury M-J, Grenier G, Bamvita J-M, Perreault M, Caron J. Variables associated with perceived unmet need for mental health care in a Canadian epidemiologic catchment area. Psychiatr Serv 2016;67:7885.

15. Sareen J, Jagdeo A, Cox BJ, et al. Perceived barriers to mental health service utilization in the United States, Ontario, and the Netherlands. Psychiatr Serv 2007;58:357-64.

16. Rens E, Dom G, Remmen R, Michielsen J, Van den Broeck K. Unmet mental health needs in the general population: perspectives of Belgian health and social care professionals. Int $\mathrm{J}$ Equity Health 2020;19:169.

17. Kroenke K, Spitzer RL, Williams JB. The PHQ-9: validity of a brief depression severity measure. J Gen Intern Med 2001;16:606-13.

18. Wittkampf KA, Naeije L, Schene AH, Huyser J, van Weert HC. Diagnostic accuracy of the mood module of the Patient Health Questionnaire: a systematic review. Gen Hosp Psychiatry 2007;29:38895.

19. Spitzer RL, Kroenke K, Williams JBW, Löwe B. A Brief Measure for Assessing Generalized Anxiety Disorder: The GAD-7. Arch Intern Med 2006;166:1092-7.

20. Löwe B, Decker O, Müller S, et al. Validation and standardization of the Generalized Anxiety Disorder Screener (GAD-7) in the general population. Med Care 2008;46:266-74.

21. Bradley KA, DeBenedetti AF, Volk RJ, Williams EC, Frank D, Kivlahan DR. AUDIT-C as a brief screen for alcohol misuse in primary care. Alcohol Clin Exp Res 2007;31:1208-17.

22. Ewing JA. Detecting Alcoholism: The CAGE Questionnaire. JAMA 1984;252:1905-7.

23. Bruffaerts R, Posada-Villa J, Al-Hamzawi AO, et al. Proportion of patients without mental disorders being treated in mental health services worldwide. Br J Psychiatry 2015;206:101-9.

24. Burgess PM, Pirkis JE, Slade TN, Johnston AK, Meadows GN, Gunn JM. Service use for mental health problems: findings from the 2007 National Survey of Mental Health and Wellbeing. Aust N Z J Psychiatry 2009;43:615-23

25. Urbanoski K, Inglis D, Veldhuizen S. Service use and unmet needs for substance use and mental disorders in Canada. Can J Psychiatry 2017;62:551-9.

26. Fernández A, Haro JM, Martinez-Alonso $M$, et al. Treatment adequacy for anxiety and depressive disorders in six European countries. Br J Psychiatry 2007;190:172-3.

27. Harris MG, Hobbs MJ, Burgess PM, et al. Frequency and quality of mental health treatment for affective and anxiety disorders among Australian adults. Med J Aust 2015;202:185-9.

28. Wang PS, Aguilar-Gaxiola S, Alonso J, et al. Use of mental health services for anxiety, mood, and substance disorders in 17 countries in the WHO world mental health surveys. Lancet 2007;370:84150.

29. Mackenzie CS, Reynolds K, Cairney J, Streiner DL, Sareen J. Disorder-specific mental health service use for mood and anxiety disorders: associations with age, sex, and psychiatric comorbidity. Depress Anxiety 2012;29:234-42. 
30. Wuthrich VM, Frei J. Barriers to treatment for older adults seeking psychological therapy. Int Psychogeriatr 2015;27:1227-36.

31. Crabb R, Hunsley J. Utilization of mental health care services among older adults with depression. J Clin Psychol 2006;62:299-312.

32. Forbes MK, Crome E, Sunderland M, Wuthrich VM. Perceived needs for mental health care and barriers to treatment across age groups. Aging Ment Health 2017;21:1072-8.

33. Giebel $\mathrm{C}$, Corcoran $\mathrm{R}$, Goodall $\mathrm{M}$, et al. Do people living in disadvantaged circumstances receive different mental health treatments than those from less disadvantaged backgrounds? BMC Public Health 2020;20:651.

34. Wang PS, Lane M, Olfson M, Pincus HA, Wells KB, Kessler RC. Twelve-month use of mental health services in the United States: results from the National Comorbidity Survey Replication. Arch Gen Psychiatry 2005;62:629-40.

35. Roy-Byrne PP, Joesch JM, Wang PS, Kessler RC. Low socioeconomic status and mental health care use among respondents with anxiety and depression in the NCS-R. Psychiatr Serv 2009;60:1190-7.

36. Evans-Lacko S, Aguilar-Gaxiola S, Al-Hamzawi A, et al. Socio-economic variations in the mental health treatment gap for people with anxiety, mood, and substance use disorders: results from the WHO World Mental Health (WMH) surveys. Psychol Med 2018;48:1560-71.

37. Jin Y, Zhu D, He P. Social causation or social selection? The longitudinal interrelationship between poverty and depressive symptoms in China. Soc Sci Med 2020;249:112848

38. Druss BG, Wang PS, Sampson NA, et al. Understanding mental health treatment in persons without mental diagnoses: results from the National Comorbidity Survey Replication. Arch Gen Psychiatry 2007;64:1196-203.

39. Manuel JI, Stebbins MB, Wu E. Gender Differences in Perceived Unmet Treatment Needs Among Persons With and Without Co-occurring Disorders. J Behav Health Serv Res 2018;45:1-12.

40. Knudsen AKS, Skogen JC, Stene-Larsen K, Gustavson K, Reneflot A. Met and unmet need for mental health care before and during the COVID-19 pandemic. Eur J Public Health 2021;ckab183

41. Gisle L, Drieskens S, Demarest S, Van der Heyden J. Geestelijke gezondheid - Gezondheidsenquête 2018. Brussels: Scienscano, 2018

42. Gisle L, Drieskens S, Demarest S, Van der Heyden J. Gebruik van alcohol - Gezondheidsenquête 2018. Brussels: Scienscano, 2018

43. Thombs BD, Kwakkenbos L, Levis AW, Benedetti A. Addressing overestimation of the prevalence of depression based on self-report screening questionnaires. CMAJ 2018;190:E44-E49

44. Levis B, Benedetti A, loannidis JPA, et al. Patient Health Questionnaire-9 scores do not accurately estimate depression prevalence: individual participant data meta-analysis. J Clin Epidemiol 2020;122:115-28

45. Smith KL, Matheson FI, Moineddin R, et al. Gender differences in mental health service utilization among respondents reporting depression in a national health survey. Health 2013;5:1561-1571 
46. Meadows G, Burgess P, Bobevski I, Fossey E, Harvey C, Liaw S-T. Perceived need for mental health care: influences of diagnosis, demography and disability. Psychol Med 2002;32:299.

47. Cohen E, Feinn R, Arias A, Kranzler HR. Alcohol treatment utilization: findings from the National Epidemiologic Survey on Alcohol and Related Conditions. Drug Alcohol Depen. 2007;86:214-21.

\section{Tables}

Table 1 and 2 are only available as a download in the Supplemental Files section.

\section{Figures}

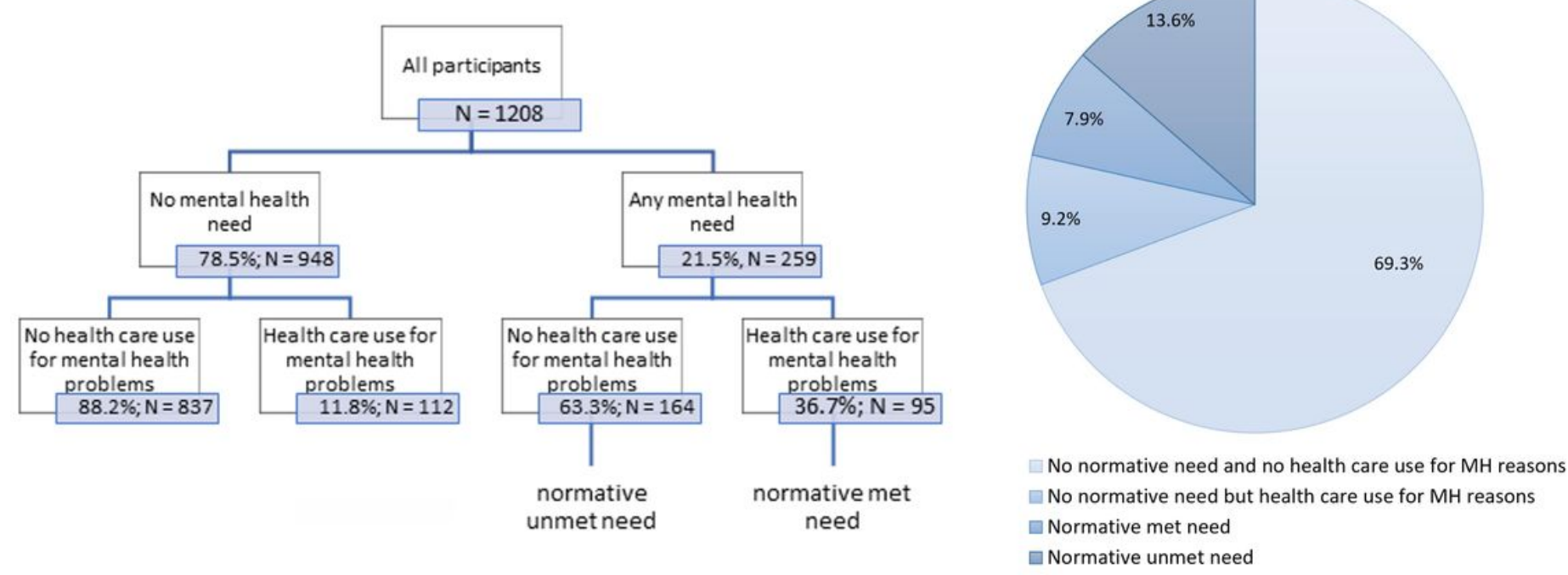

\section{Figure 1}

Classification and population distribution of normative (unmet) needs for mental health care. 

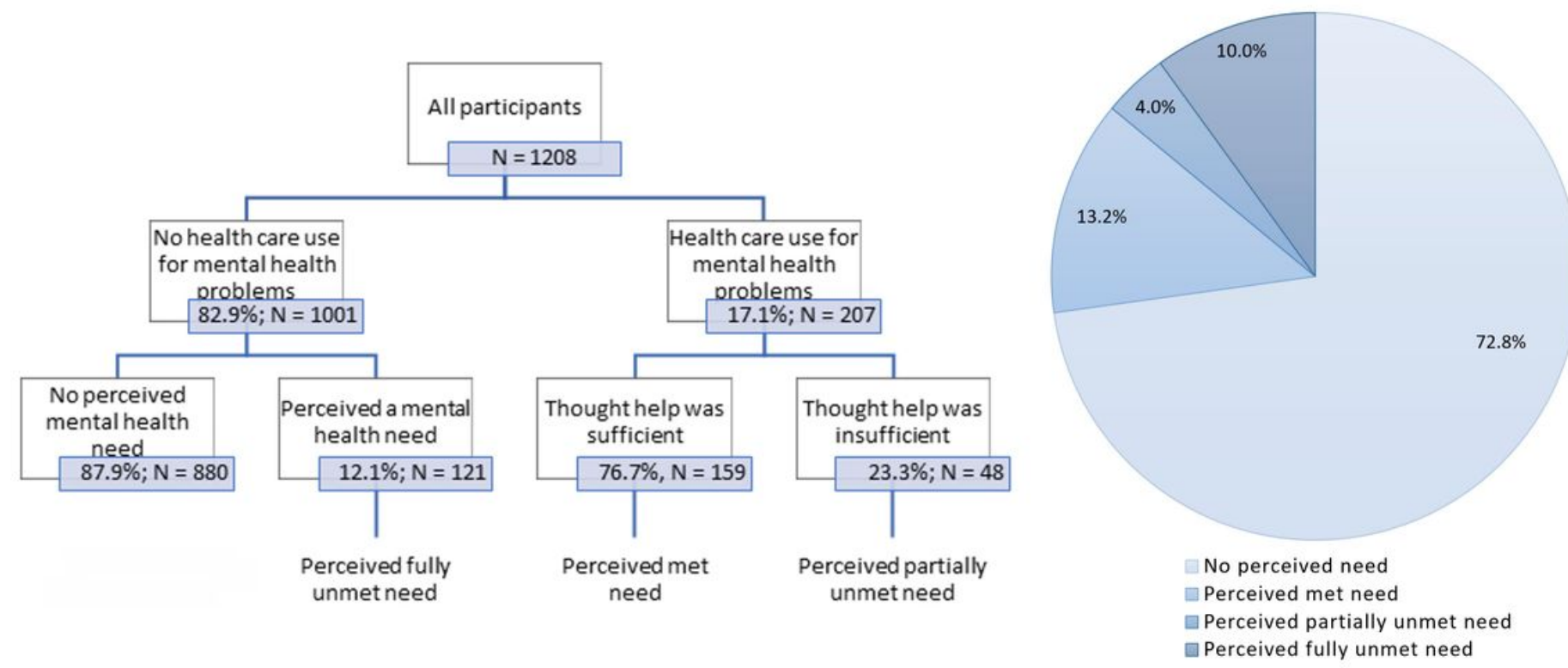

Figure 2

Classification and population distribution of perceived (unmet) needs for mental health care.

\section{Supplementary Files}

This is a list of supplementary files associated with this preprint. Click to download.

- Table1.docx

- Table2.docx 